\title{
Behavioral Recovery in MPTP-Treated Monkeys: Neurochemical Mechanisms Studied by Intrastriatal Microdialysis
}

\author{
Sabrina Boulet, ${ }^{1,2,3}$ Stéphanie Mounayar, ${ }^{4,5}$ Annie Poupard, ${ }^{1,2,3}$ Anne Bertrand, ${ }^{1,2,3}$ Caroline Jan, ${ }^{4,5}$ \\ Mathias Pessiglione, ${ }^{4,5}$ Etienne C. Hirsch, ${ }^{4,5}$ Claude Feuerstein, ${ }^{1,2,3}$ Chantal François, ${ }^{4,5}$ Jean Féger, ${ }^{4,5}$ Marc Savasta, ${ }^{1,2,3 *}$ \\ and Léon Tremblay ${ }^{6 *}$ \\ ${ }^{1}$ Institut National de la Santé et de la Recherche Médicale, Unité 836, Grenoble Institut des Neurosciences, Dynamique des Réseaux Neuronaux du \\ Mouvement and ${ }^{2}$ Centre Hospitalier Universitaire de Grenoble, Grenoble F-38043 Cedex 09, France, ${ }^{3}$ Université Joseph Fourier, Grenoble F-38041 Cedex \\ 09, France, ${ }^{4}$ Institut National de la Santé et de la Recherche Médicale, Unité 679 and ${ }^{5}$ Université Pierre et Marie Curie-Paris 6, Institut Fédératif de \\ Recherche de Neurosciences Unité Mixte de Recherche (UMR) S679, Paris F-75013, France, and ${ }^{6}$ Institut des Neurosciences Cognitives-UMR 5229, Centre \\ National de la Recherche Scientifique-Université de Lyon 1, Bron F-69675 Cedex, France
}

Parkinson's disease (PD) patients express motor symptoms only after $60-80 \%$ striatal dopamine (DA) depletion. The presymptomatic phase of the disease may be sustained by biochemical modifications within the striatum. We used an appropriate specific 1-methyl-4phenyl-1,2,3,6-tetrahydropyridine (MPTP) monkey model (Mounayar et al., 2007) to study the compensatory mechanisms operating in recovery from PD motor symptoms. We assessed the levels of DA and its metabolites (DOPAC, homovanillic acid), GABA, glutamate (Glu), serotonin (5-HT) and its metabolite (5HIAA) by repeated intracerebral microdialysis in awake animals before exposure to MPTP during full expression of the motor symptoms induced by MPTP and after recovery from these symptoms. Measurements were obtained from two functionally and anatomically different striatal areas: the associative-limbic territory and sensorimotor territory. Animals with motor symptoms displayed an extremely large decrease in levels of DA and its metabolites and an increase in Glu and GABA levels, as reported by other studies. However, we show here for the first time that serotonin levels increased in these animals. We found that increases in DA levels in the sensorimotor and/or associative-limbic territory and high levels of 5-HT and of its metabolite, 5HIAA, were associated with recovery from motor symptoms in this model. Determining whether similar changes in DA and 5-HT levels are involved in the compensatory mechanisms delaying the appearance of motor symptoms in the early stages of PD might make it possible to develop new treatment strategies for the disease.

Key words: Parkinson's disease; recovery; MPTP; primate; microdialysis; serotonin

\section{Introduction}

The motor symptoms of Parkinson's disease (PD), namely resting tremor, rigidity, and bradykinesia, are expressed when striatal dopamine (DA) depletion exceeds 60-80\% (Bernheimer et al., 1973). The existence of such a threshold suggests that compensatory mechanisms (Zigmond et al., 1990) compensate for initial dopaminergic dysfunction, delaying the appearance of clinical symptoms.

\section{Received July 23, 2008; accepted Aug. 15, 2008.}

This work was supported by the Institut National de la Santé et de la Recherche Médicale, Région Rhône-Alpes (Cluster ${ }^{\circ}{ }^{\circ 11)}$, the National Parkinson Foundation (Miami, Florida), and the Association France Parkinson. S.B. was supported by the French Ministry of Research and New Technologies and the Association France Parkinson; S.M. was supported by the Association France Parkinson and the Fondation pour la Recherche Médicale (France). We thank Nicolas Bruet for technical assistance and Jeffrey Hollerman for helpful suggestions on data analysis and English correction.

*M.S. and L.T. contributed equally to this work.

Correspondence should be addressed to Marc Savasta, Grenoble Institut des Neurosciences, Institut National de la Santé et de la Recherche Médicale U836, Dynamique des Réseaux Neuronaux du Mouvement, Grenoble F-38043 Cedex 09, France. E-mail: Marc.Savasta@ujf-grenoble.fr.

D01:10.1523/JNEUROSCI.3465-08.2008

Copyright $\odot 2008$ Society for Neuroscience $\quad$ 0270-6474/08/289575-10\$15.00/0
Many neurochemical data are available on PD patients. However, most concern striatal dysfunction after the onset of motor symptoms and are limited to determinations of the concentrations of DA, glutamate (Glu) and GABA only. It is thus well established in humans (Hornykiewicz, 1975, 1998; Agid et al., 1987) and in animal models of the disease, such as 6-hydroxydopamine (6-OHDA)-lesioned rats (Hefti et al., 1980; Schwarting and Huston, 1996) and 1-methyl-4-phenyl-1,2,3,6tetrahydropyridine (MPTP)-lesioned monkeys (Elsworth et al., 1989; Skirboll et al., 1990; McCallum et al., 2006), that the motor symptom state of PD is characterized by a large decrease in levels of DA and its metabolites and an increase in DOPAC/DA and homovanillic acid (HVA)/DA ratios. Increases in striatal concentrations of Glu and GABA have also been reported in humans (Kish et al., 1988; Hornykiewicz, 2001), 6-OHDA rats (Lindefors and Ungerstedt, 1990; Meshul et al., 1999; Bruet et al., 2003), and MPTP monkeys (Robinson et al., 2003). The few studies on compensatory mechanisms have been performed in MPTP monkeys and cats recovering spontaneously from PD motor symptoms. They revealed that spontaneous recovery is associated with an 
increase in striatal DA release (Rose et al., 1989; Schneider and Rothblat, 1991; Schneider et al., 1994) and turnover (Petzinger et al., 2006) with respect to the symptomatic state. However, the neurochemical basis of PD compensatory mechanisms, including the potential role of neurotransmitters other than dopamine, remains unclear.

We investigated striatal biochemical changes related to compensation, differentiating these alterations from those associated with motor symptoms. We worked on a specific MPTP monkey model displaying complete recovery after the cessation of MPTP exposure (Mounayar et al., 2007). We performed repeated intracerebral microdialysis in awake animals, monitoring neurochemical changes during the presymptomatic phase, at the peak of motor symptom expression, and after complete recovery. Microdialysis was performed simultaneously in two different striatal territories, the sensorimotor territory and the associative-limbic territory, to take into account the anatomical and functional heterogeneity of the striatum. We focused on levels of DA and its metabolites, DOPAC and HVA, GABA and Glu, all of which are known to be modified by MPTP treatment, and levels of serotonin and its metabolite, 5HIAA. We previously showed that stable motor symptom expression in this animal model is associated with a decrease in the number of striatal serotonin fibers, whereas recovery is associated with an increase in the number of these fibers (Mounayar et al., 2007), suggesting a role for serotonin neurotransmission in motor recovery.

\section{Materials and Methods}

Animals. Five male vervet monkeys (Cercopithecus aethiops) between 4 and 6 years old (young adults) and weighing $4-7 \mathrm{~kg}$, provided by the Barbados Primate Research Centre (Farley Hill, Barbados, West Indies), were used in this study. These monkeys were cared for and treated in strict accordance with National Institutes of Health guidelines (1996), the European Community Council Directive of 1986 (86/609/EEC), and the recommendations of the French National Committee (87/848).

Surgery. Surgery was performed under deep anesthesia induced by ketamine/atropine injection and maintained by fluothane inhalation. The anterior commissure (AC) and posterior commissure (PC) were located during surgery by ventriculography. A rectangular opening was made in the skull such that the dura remained intact. A rectangular stainless steel recording chamber $(30 \times 26 \times 21 \mathrm{~mm}$; Unimécanique $)$ was fixed over this hole with surgical screws and dental acrylic resin. The chamber was positioned stereotactically in the horizontal plane parallel to the AC-PC axis, with the anterior-posterior center on the AC and 2 $\mathrm{mm}$ over the right hemisphere. Two stainless steel cylinders for head fixation were also cemented in place in front of and behind the chamber.

Experimental schedule. The monkeys were trained to sit in a primate chair for manipulation, and their spontaneous behavior was quantified (Mounayar et al., 2007). Surgery was followed by a period of retraining to ensure that the monkey's performance remained accurate and stable. At the end of this period, behavioral data were collected over $5 \mathrm{~d}$ to characterize the normal state. Animals were then subjected to progressive MPTP intoxication (injections $4-5 \mathrm{~d}$ apart). All monkeys received repeated intramuscular injections of $0.4-0.6 \mathrm{mg} / \mathrm{kg}$ MPTP (SigmaAldrich) under light anesthesia $(0.1 \mathrm{ml} / \mathrm{kg}$ ketamine, $0.05 \mathrm{ml} / \mathrm{kg}$ atropine). Injections were repeated until the desired motor score was achieved. The total dose given to each monkey varied between 1.3-2.5 $\mathrm{mg} / \mathrm{kg}$. Once MPTP injections were stopped, the animals recovered spontaneously from their motor symptoms. At the end of the experiment, monkeys were deeply anesthetized with an overdose of barbiturate and transcardially perfused with saline followed by fixative solution.

Behavioral analysis. The progression of parkinsonian motor symptoms was evaluated, using the rating scale proposed by Schneider and Kovelowski (1990) (Mounayar et al., 2007).

Microdialysis experiment. Microdialysis was performed simultaneously in two different striatal territories in each awake animal: the sensorimotor territory (right posterior striatum) and the associativelimbic territory (left anterior striatum). Each monkey underwent three microdialysis sessions, corresponding respectively to the normal state, the symptomatic state (highest motor score), and the recovery state over a 3 month period. A supplementary dialysis session was performed the day after the third session, within the associative-limbic territory in the right hemisphere, in which no previous dialysis had been carried out. The neurochemical data obtained for this new site were compared with those obtained the day before, to estimate the influence of repeated dialysis on neurotransmitter concentrations.

The microdialysis probes used for these experiments were made on site. They consisted of a concentric arrangement of a stainless-steel tube (length, $90 \mathrm{~mm}$; 24 gauge; Phymep), and polyethylene tubing (length, 10 $\mathrm{mm}$; external diameter (ED), $1.09 \mathrm{~mm}$; internal diameter (ID), $0.38 \mathrm{~mm}$; Biotroll Diagnostic), into which we placed a piece of silica tubing (length, $120 \mathrm{~mm}$; ED, $150 \mu \mathrm{m}$; ID, $75 \mu \mathrm{m}$; Phymep). The silica tubing extended beyond the distal end of the steel tube and was covered by an AN69 tubular dialysis membrane (acrylonitrile and sodium methallyl sulfonate copolymer, cutoff $40 \mathrm{kDa}$; Hospal Industrie) sealed at the bottom with epoxy glue. The length of the silica tubing and of the dialysis membrane was adapted according to the monkey brain nucleus studied: $3 \mathrm{~mm}$ for the anterior and posterior striatum. Before use, microdialysis probes were tested in vitro in a standard solution of amino acids and catecholamines; their flow was checked and their efficiency calculated (Tossman and Ungerstedt, 1986): Glu, $21 \pm 0.8 \%$; GABA, $25.3 \pm 1.2 \%$; DA, $12.4 \pm 1.9 \%$; 5 -HT, $13.2 \pm 1.6 \%$. Probes with an abnormal flow or efficiency were discarded so that all probes used in the study presented homogeneous technical characteristics.

The perfusion liquid flowed out of the distal end of the steel tube, passing proximally between the tube and the membrane (Tossman and Ungerstedt, 1986). The probes were perfused with artificial CSF [(in nм) $149 \mathrm{NaCl}, 2.8 \mathrm{KCl}, 1.2 \mathrm{MgCl}_{2}, 1.2 \mathrm{CaCl}_{2}, 5.4$ glucose, $\mathrm{pH} 7.3$ ) at a flow rate of $2 \mu \mathrm{l} / \mathrm{min}$ (de Lange et al., 2000; Khan and Shuaib, 2001) using a micropump (CMA/100; Phymep).

The head of the animal was fixed in place and a grid system $(22 \times 14$ $\mathrm{mm}$; Unimécanique) was placed in the recording chamber. This system, the dimensions of which were adapted to the size of the recording chamber, was used to locate the two dialysis probes precisely, at $4 \mathrm{~mm}$ from the $\mathrm{AC}$ in the anterior striatum and $4 \mathrm{~mm}$ behind the $\mathrm{AC}$ in the posterior striatum, making it possible to use precisely the same sites for all three dialyses. The dialysis probes were then lowered into the two striatal territories through cannula guides (21G; Phymep) using a vertical microdriver. Dialysates were collected from awake animals at 15 min intervals. The first six fractions were discarded to avoid the effects of parenchymal disturbance and to ensure that an approximate steady-state level was reached. Fractions were then collected over a period of $\sim 3.5-4 \mathrm{~h}$ during which time the animals remained quiet. The dialysates were automatically collected with a refrigerated autosampler (Microsampler 820; Univentor, Phymep) and stored at $-80^{\circ} \mathrm{C}$ until analysis.

Neurotransmitter assays. Concentrations of Glu, GABA, dopamine, DOPAC, HVA, serotonin, and 5HIAA were determined for each sample.

Amino acid assay. Levels of Glu and GABA in dialysates were determined by high-performance liquid chromatography (HPLC) coupled with laser-induced fluorimetric detection. Briefly, $2 \mu$ l of sample or standard was derivatized with naphthalene-2,3-dicarboxaldehyde. A refrigerated autoinjector (Triathlon; Polymer Laboratories) was used to inject the sample into a Symmetry Shield-C18 reverse-phase column $(100 \times$ $2.1 \mathrm{~mm}, 3.5 \mu \mathrm{m}$ particle size) (Waters). The mobile phase consisted of $0.04 \mathrm{M} \mathrm{NaH}_{2} \mathrm{PO}_{4}, \mathrm{pH}$ 6, with a 3-50\% acetonitrile gradient, delivered at a flow rate of $0.35 \mathrm{ml} / \mathrm{min}$ by two Shimadzu LC-10AT pumps. Amino acid peaks were identified based on retention time. Extracellular amino acid levels were estimated by determining the ratio of the peak area for each amino acid to that for the corresponding external standard (analytical software class LC10; Shimadzu. The running time for each determination was $12 \mathrm{~min}$.

Catecholamine assay. Levels of dopamine, DOPAC, HVA, 5-HT, and 5HIAA were determined by HPLC coupled with electrochemical detection. The system consisted of a pump (LC-10 AD; Shimadzu), a refrigerated automatic injector (Famos; Dionex), a reverse-phase Hypersil RP 18 
Table 1. Behavioral data and levels of dopaminergic degeneration in the $\mathrm{SN}$ and striatum

\begin{tabular}{|c|c|c|c|c|c|c|c|}
\hline \multirow[b]{2}{*}{ Monkey } & \multirow[b]{2}{*}{ Total dose of MPTP $(\mathrm{mg} / \mathrm{kg})$} & \multirow[b]{2}{*}{ Maximal score } & \multirow[b]{2}{*}{ Final score } & \multirow[b]{2}{*}{ Recovery time (days) } & \multirow[b]{2}{*}{ SN denervation(\%) } & \multicolumn{2}{|c|}{ Striatal denervation (\%) } \\
\hline & & & & & & Sensorimotor & Mean associative-limbic (associative-limbic) \\
\hline CA 21 & 2.3 & 9 & 0 & 20 & 31.7 & 58 & $21.5(29-14)$ \\
\hline CA 34 & 1.4 & 12 & 0 & 24 & 68.4 & 85 & $79(79-79)$ \\
\hline CA 23 & 2.5 & 19 & 0 & 33 & 73.6 & 81 & $77.5(86-69)$ \\
\hline CA 33 & 2.1 & 19 & 0 & 17 & 56.9 & 76 & $64(66-62)$ \\
\hline CA 37 & 1.3 & 22 & 2 & 45 & 78.8 & 91 & $74.5(86-63)$ \\
\hline
\end{tabular}

column (Aquasil, $150 \times 1 \mathrm{~mm}, 3 \mu \mathrm{m}$; ThermoHypersil), and an electrochemical detector (Decade; Antec) equipped with an amperometric cell (VT-03; Antec). Chromatograms were collected and treated with an integrator (CLAS VP; Shimadzu). The mobile phase consisted of sodium phosphate buffer $\left(50 \mathrm{~mm} \mathrm{NaH}_{2} \mathrm{PO}_{4}\right)$, 1-octanesulfonic acid (1.7 mM), $\mathrm{Na}_{2}$ EDTA $(200 \mu \mathrm{m})$ with $5 \%$ of acetonitrile at $\mathrm{pH} 3$ for DA metabolites, and $\mathrm{pH} 4$ for DA and 5-HT. The mobile phase was delivered by the pump at a flow rate of $60 \mu \mathrm{l} \cdot \mathrm{min}^{-1}$. The oxidation potential of the electrochemical cell was $+650 \mathrm{mV}$. A volume of $5 \mu \mathrm{l}$ was injected, and the running time for each determination was $35 \mathrm{~min}$.

Reproducibility. We checked the relevance of repeated dialysis at the same site by comparing the neurotransmitter concentrations obtained during the third dialysis with those obtained during supplementary dialysis on the contralateral side of the striatum at a previously untested site. This control was performed for three animals (CA 23, CA 34, and CA 37).

Histological analysis. The fixed brains were sectioned in the frontal plane and processed for immunohistochemical procedures (Jan et al., 2000). We stained for calbindin ( $\mathrm{Cb})$, tyrosine hydroxylase (TH), and 5 -HT on regularly spaced sections from each monkey. Dopaminergic denervation was then studied by immunocytochemical localization of $\mathrm{TH}$. TH-positive cells were counted in the substantia nigra (SN). Striatal dopaminergic and serotoninergic innervation was evaluated by determining optical density or by counting $\mathrm{TH}$-positive fibers in the sensorimotor, associative, and limbic functional territories. Cresyl violet staining and $\mathrm{Cb}$ and GFAP immunoreactivity were used to identify the probe tracks and to assess the cell damage and inflammatory reactions potentially induced by three successive dialysis sessions at the same site.

Immunocytochemical techniques. For $\mathrm{Cb}$ immunostaining, sections were incubated with mouse anti-Cb antibody (1:2000 dilution; SigmaAldrich), followed by a secondary biotinylated antibody (1:200 dilution, goat anti-mouse IgG; Vector Laboratories). Similar methods were used for TH immunostaining but with a primary mouse anti-TH antibody (1:500 dilution; Incstar) followed by a secondary biotinylated antibody (1:250 dilution, goat anti-mouse IgG; Vector Laboratories) (François et al., 1999).

5-HT immunostaining was performed using a primary rabbit anti5-HT antibody (1:10,000 dilution; Diasorin) followed by a secondary biotinylated antibody (1:200 dilution, goat anti-rabbit, IgG; SigmaAldrich). Finally, GFAP immunostaining was performed with a primary rabbit anti-GFAP antibody (1:4000 dilution; Sigma-Aldrich), followed by a secondary biotinylated antibody (1:200 dilution, goat anti-rabbit IgG; Sigma-Aldrich).

In all cases, immunostaining was visualized using avidin-biotinperoxidase complex (ABC standard kit, 1:125 in PBS; Vector Laboratories) and diaminobenzidine (Sigma-Aldrich).

Quantification methods. TH-positive cells in the substantia nigra pars compacta $(\mathrm{SNc})$ were plotted on nine frontal sections at $1 \mathrm{~mm}$ intervals, using a computer-assisted microscope system (Mercator; Explora Nova). The total number of cells was estimated after correction by the Abercrombie method (1946), and neuronal loss was evaluated by comparison with control values for intact vervet monkeys (Jan et al., 2000; Mounayar et al., 2007).

For the quantification of TH- and 5-HT-labeled fibers, functional territories of the striatum were delineated rostrally $(\mathrm{AC}+3.95$; limbic and associative territories) and caudally ( $\mathrm{AC}-4.55$; sensorimotor territory). Attributable to the high density of TH- and 5-HT-positive fibers in control monkeys, dopaminergic and serotoninergic innervation was evaluated by measuring the optical density of immunostaining with an image analysis system (Mercator; Explora Nova). We also visually quantified individual fibers in the sensorimotor territory, for MPTP-treated monkeys only (Mounayar et al., 2007).

Data and statistical analysis. For microdialysis experiments, basal levels correspond to the mean concentration of the 15 samples during the first dialysis, before the animals were treated with MPTP. These basal levels were defined as $100 \%$, and neurotransmitter concentrations in the motor symptom state or after recovery are expressed as percentages of this value. The effects of MPTP injections and recovery on extracellular Glu, GABA, DA, DOPAC, HVA, 5-HT, and 5HIAA levels were analyzed by Wilcoxon analysis. Values of $p<0.05$ were considered statistically significant.

For each neurotransmitter studied, Pearson's correlation analysis was performed in each territory and at each motor state. These correlation analysis were done for motor behavior and dopaminergic and 5-HT denervation, estimated on the basis of optical density or fiber counts. Finally, the relationships between neurotransmitters were investigated by cross-correlation analysis.

\section{Results}

\section{Behavioral data}

No correlation was found between the motor score and the cumulative dose of MPTP received by each animal (Table 1). All animals totally recovered from their motor symptoms after MPTP injections stopped, with the exception of CA 37 (motor score of 2 when killed). The time required for complete recovery increased with maximal motor score in all monkeys except CA 33. This monkey recovered rapidly (17 d) from severe motor symptoms (score 19). Counts of residual dopaminergic neurons in the SNc and fibers in the striatum indicated that degeneration was generally proportional to motor symptom severity, with apparent individual exceptions. Dopaminergic neuron or fiber loss, for example, was smallest in the monkey presenting the mildest motor symptoms (CA 21).

\section{Histological control}

Location of microdialysis probes

In the postero-lateral part of the striatum, the probes were homogeneously implanted in the sensorimotor territory (no immunostaining for calbindin) (Fig. 1D), with slight variations in dorso-ventral positioning (Fig. $1 C$ ). In the associative-limbic territory, all of the probes were located at the junction of these two striatal territories (Fig. 1A), both displaying positive calbindin immunostaining (Fig. $1 B$ ). However, differences in laterality were observed with the probes for CA 34 and 37 which were located more medially (in the ventral part of the caudate nucleus) than those for CA 21, 23, and 33 (located in the putamen).

The site of the supplementary dialysis sessions, within the contralateral hemisphere, was almost identical to that used for the first three assessments for CA 23 and 34 but was slightly more lateral for CA 37.

\section{Safety of repeated microdialysis for cerebral tissue}

The enlargements of Figure 1, $B$ and $D$, clearly show that three successive passages of dialysis probes at the same site of the sensorimotor or associative-limbic striatal territory resulted in cel- 

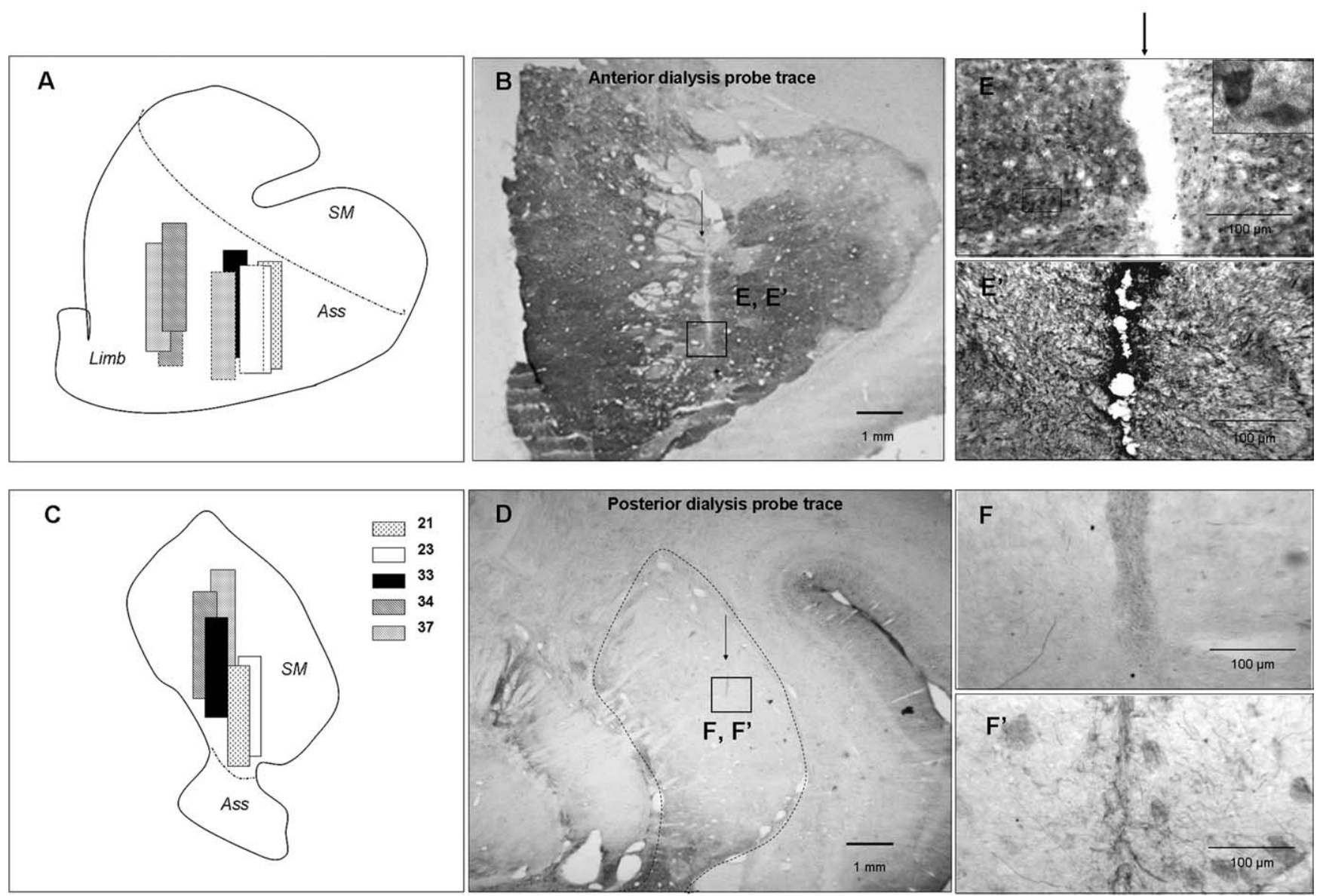

Figure 1. Histological controls of dialysis probe location. $\boldsymbol{A}, \boldsymbol{C}$, Schematic representation of dialysis probe location in the associative-limbic $(\boldsymbol{A})$ and sensorimotor $(\boldsymbol{C})$ striatal territories in the five monkeys studied. Solid lines indicate the first, second, and third dialyses, and dotted lines correspond to the fourth supplementary dialysis. SM, Sensorimotor striatum; Ass, associative striatum; Limb, limbic striatum. $\boldsymbol{B}, \boldsymbol{D}$, Locations of dialysis probes in the associative-limbic $(\boldsymbol{B})$ and sensorimotor $(\boldsymbol{D})$ striatum on a calbindin-immunostained coronal brain section. Scale bar, $1 \mathrm{~mm}$. $\boldsymbol{E}-\boldsymbol{F}^{\prime}$, Dialysis probe traces at a higher magnification $(6.5 \times)$ after immunostaining for calbindin $(\boldsymbol{E}, \boldsymbol{F})$ or GFAP $\left(\boldsymbol{E}^{\prime}, \boldsymbol{F}\right)$ in the associative-limbic $(\boldsymbol{E}, \boldsymbol{E})$ or sensorimotor $(\boldsymbol{F}, \boldsymbol{F})$ territory. Scale bar, $100 \mu \mathrm{m}$.

lular damage no more than $100 \mu \mathrm{m}$ in diameter (Fig. $1 \mathrm{E}, F$ ). This cell loss appeared to be limited to the dialysis probe track, as neuronal cell bodies were visible adjacent to this track (Fig. 1E). Furthermore, examinations of $\mathrm{TH}$ - and 5-HT-immunostained sections showed dopaminergic and serotoninergic fibers in the immediate vicinity of the track in both the sensorimotor and associative-limbic territories after MPTP treatment and recovery from motor symptoms (Fig. 2). Finally, GFAP immunostaining showed that repeated microdialysis had induced a mild glial reaction limited to the immediate vicinity of the track (Fig. $1 E^{\prime}, F^{\prime}$ ).

\section{Basal levels of neurotransmitters and variability of measurements}

The first dialysis was performed before the first MPTP injection and provided basal extracellular concentrations for each neurotransmitter studied in the two striatal territories. Dopamine concentration was four times higher in the sensorimotor than in the associative-limbic territory $(n=5 ; p<0.01)$ (Table 2). In contrast, for DA metabolites and all other neurotransmitters, concentrations were significantly higher in the associative-limbic than in the sensorimotor territory $(p<0.01)$ (Table 2) by factors of $5,2,2.5,1.5$, and 1.5 for DOPAC, HVA, 5-HT, glutamate, and GABA levels, respectively (Table 2). DOPAC/DA and HVA/DA ratios were also higher in the associative-limbic territory than in the sensorimotor territory $(\times 15, p<0.05$; $\times 6, p<0.01$, respectively), whereas the opposite pattern was observed for 5HIAA/ 5-HT ratio $(\times 5)($ Table 2$)$.

After the third dialysis session (after recovery), neurotransmitter concentrations were determined during the supplementary dialysis session in the associative-limbic territory on the contralateral side. The concentrations measured differed by no more than $20 \%$ between the two hemispheres, except for DOPAC and glutamate concentrations, which were 199 and 75\% higher, respectively, in the contralateral limbic-associative striatum than at the sites used for the first three measurements.

\section{Changes in sensorimotor and associative-limbic neurotransmitter levels associated with the expression of motor symptoms and recovery}

Effects on dopamine levels

In the sensorimotor striatum, DA levels were much lower in the symptomatic state than in the normal state $(-96.7 \pm 1.6 \% ; p<$ 0.01) (Fig. 3A). This decrease was correlated with the level of dopaminergic denervation, estimated by determining the optical density of immunostaining for TH $(r=0.89$; $p<0.05)$ and the DA transporter (DAT) $(r=0.92 ; p<0.05)$. After recovery, DA levels remained much lower than those in the normal state 
Sensorimotor
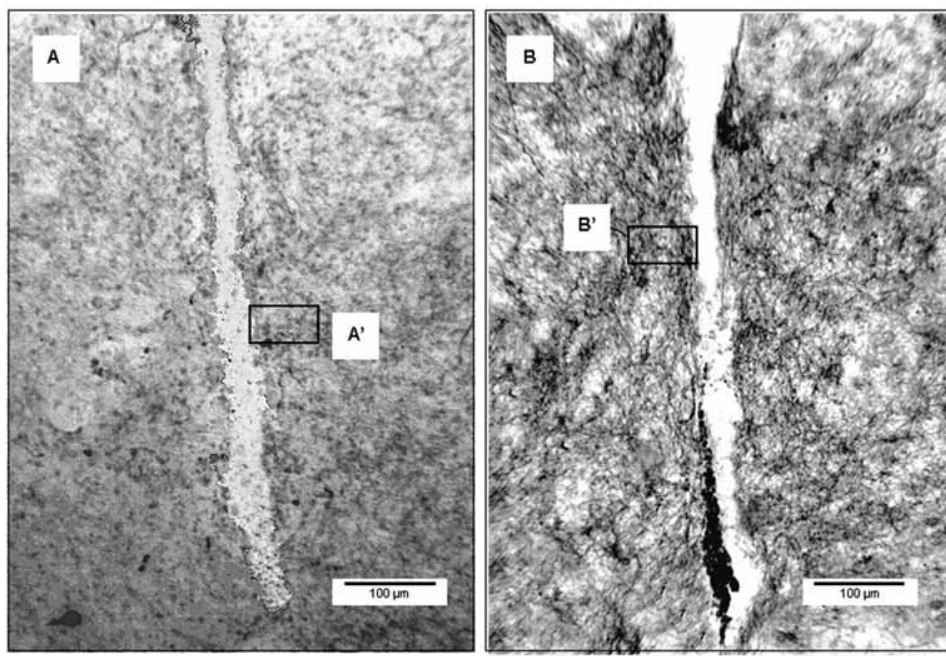
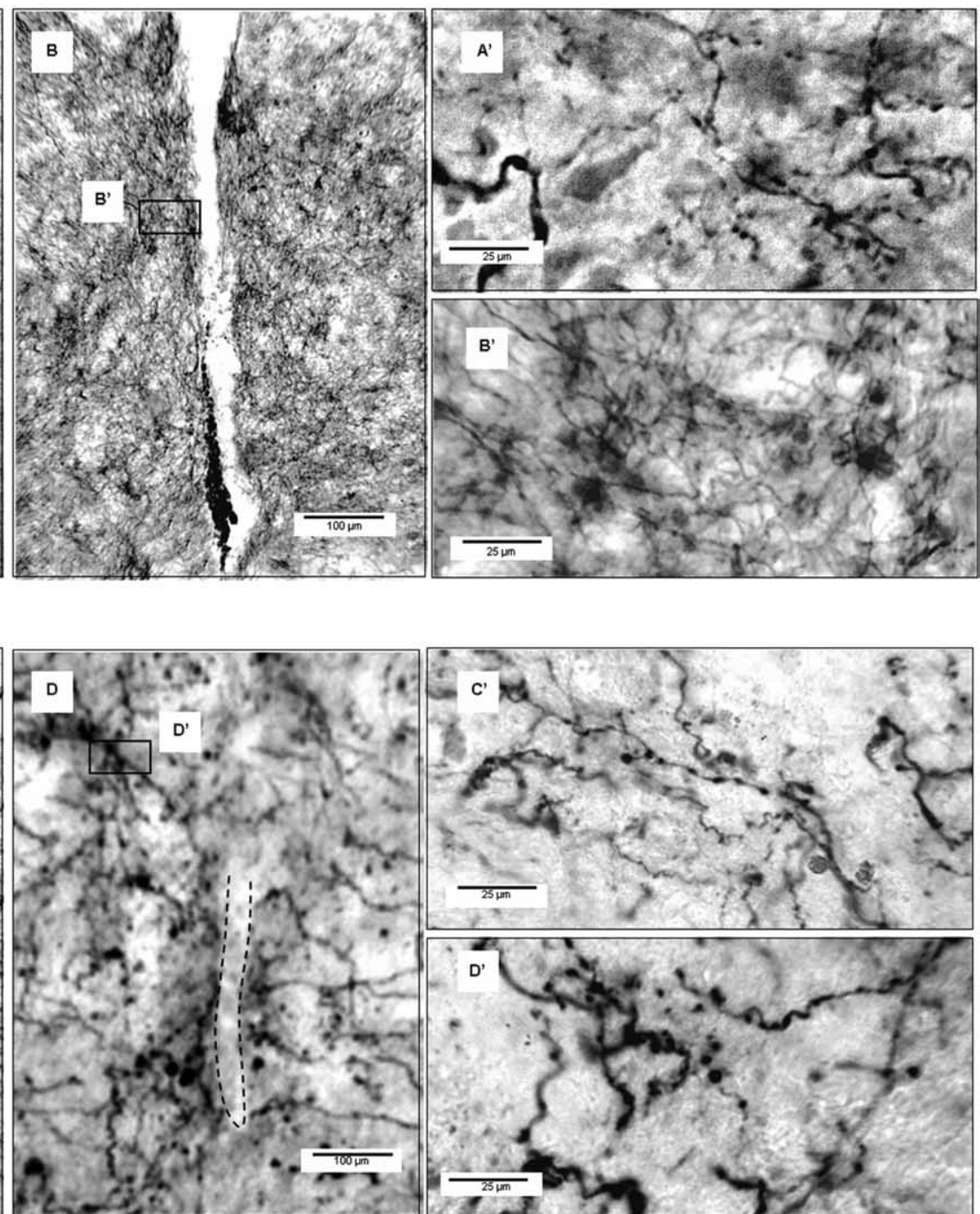

Figure 2. Tissue integrity in the vicinity of dialysis sites. $\boldsymbol{A}-\boldsymbol{D}^{\prime}, \mathrm{TH}\left(\boldsymbol{A}-\boldsymbol{B}^{\prime}\right)$ and $5-\mathrm{HT}\left(\boldsymbol{C}-\boldsymbol{D}^{\prime}\right)$ immunostaining on coronal brain sections for the sensorimotor $\left(\boldsymbol{A}, \boldsymbol{A}^{\prime}, \boldsymbol{C}, \boldsymbol{C}^{\prime}\right)$ and associative-limbic $(\boldsymbol{B}$, $\left.\boldsymbol{B}^{\prime}, \boldsymbol{D}, \boldsymbol{D}^{\prime}\right)$ territories. $\boldsymbol{A}^{\prime}, \boldsymbol{B}^{\prime}, \boldsymbol{C}^{\prime}, \boldsymbol{D}^{\prime}$, Enlargements (40X; scale bar, $\left.25 \mu \mathrm{m}\right)$ of $\boldsymbol{A}, \boldsymbol{B}, \boldsymbol{C}$, and $\boldsymbol{D}$, respectively $(6.5 \times$; scale bar, $100 \mu \mathrm{m})$.

Table 2. Extracellular concentrations ( $\mathrm{pg} / \mu \mathrm{l})$ of dopamine, DOPAC, HVA, glutamate, GABA, 5-HT, DOPAC/DA, HVA/DA, and 5HIAA/5HT concentration ratios in the sensorimotor and associative-limbic striatum of normal monkeys (before MPTP injection), with statistical analysis

\begin{tabular}{lcc}
\hline Neurotransmitter & Sensorimotor & Associative-limbic \\
\hline DA & $4.16 \pm 0.71$ & $1.01 \pm 0.29^{* *}$ \\
DOPAC & $10.71 \pm 3.13$ & $52.19 \pm 19.02^{* *}$ \\
HVA & $399.39 \pm 56.24$ & $782.96 \pm 116.26^{* *}$ \\
5HT & $0.17 \pm 0.12$ & $0.42 \pm 0.19^{* *}$ \\
Glu & $100.33 \pm 35.85$ & $161.01 \pm 78^{*}$ \\
GABA & $4.49 \pm 1.21$ & $7.34 \pm 2.19^{* *}$ \\
Ratio & & \\
$\quad$ DOPAC/DA & $2.77 \pm 0.85$ & $41.13 \pm 11.72^{* *}$ \\
HVA/DA & $107.28 \pm 18.95$ & $622.63 \pm 25.03^{* *}$ \\
5HIAA/5HT & $713.95 \pm 211.87$ & $150.42 \pm 39.92^{* *}$ \\
\hline
\end{tabular}

${ }^{*} p<0.05 ;{ }^{* *} p<0.01$.

$(-88.8 \pm 6.62 \% ; p<0.01)$ (Fig. $3 A)$ and not significantly different from those in the symptomatic state.

The decrease in DA levels observed in the associative-limbic territory of symptomatic monkeys (53.2 $\pm 35.5 \%)$ (Fig. $3 A$ ) was smaller than that in the sensorimotor territory. Once the monkeys had recovered from their motor symptoms, DA concentration returned to basal levels $(95.9 \pm 51.2 \%)$.

\section{Effects on DOPAC levels}

DOPAC levels were severely and significantly decreased in both striatal territories $(p<0.01)$ (Fig. $3 B)$ in the symptomatic state. DOPAC concentration was significantly higher after recovery than in the motor symptom state only in the associative-limbic territory $(p<0.01)$ (Fig. 3B).

\section{Effects on HVA levels}

A significant, strong decrease in HVA levels was measured in both striatal territories $(p<0.01)$ (Fig. $3 C$ ) in the symptomatic state. This decrease was positively correlated with the loss of $\mathrm{TH}$ positive fibers in the sensorimotor territory $(r=0.94 ; p<0.05)$ (see Fig. $6 B$ ). HVA levels after recovery were significantly higher than those in the symptomatic state for both territories but remained significantly lower than basal levels (Fig. 3C).

Effects on HVA/DA and DOPAC/DA ratio

The HVA/DA ratio was markedly increased in the sensorimotor striatal territory in the symptomatic state $(p<0.01)$ (Fig. $3 D)$. 
When the animals had totally recovered, this ratio increased further, to a level significantly higher than that in the symptomatic state $(p<0.01)$ (Fig. $3 D)$. Motor symptom expression had no significant effect on the HVA/DA ratio (127.6 \pm $72.9 \%)$ in the associative-limbic territory (Fig. 3D). After recovery from motor symptoms, this ratio increased to a level significantly higher than that in the normal state $(713.3 \pm 520.2 \%$; $p<0.01)$ (Fig; $3 \mathrm{D})$ but not significantly different from that in the symptomatic state.

The effects of motor symptom expression and recovery on the DOPAC/DA ratio varied more between territories. In the motor symptom state, the DOPAC/DA ratio was higher in the sensorimotor territory and significantly lower in the associative-limbic striatum $(p<0.01)$ than in the normal state. After recovery from motor symptoms, the DOPAC/DA ratio was higher than in the motor symptom state, reaching values greater than those for the normal state in both territories $(379.9 \pm 52.2 \%, p<0.01 ; 178.7 \pm$ $84.7 \%)$.

\section{Effects on serotonin levels}

A large increase in 5-HT concentration was measured in the sensorimotor striatum in the symptomatic state $(510.9 \pm 119 ; p<0.01)$ (Fig. $4 A$ ). After recovery from motor symptoms, 5-HT levels remained high, well above those in the normal state $(372 \pm 95.3 ; p<0.01)$ (Fig. $4 A)$.

5 -HT levels were also higher in the associative-limbic territory in the symptomatic state than in the normal state, but this increase was less pronounced than that in the sensorimotor territory $(198.7 \pm 57.3 ; p<0.01)$ (Fig. $4 A$ ). This increase was negatively correlated with the number of residual TH-positive fibers $(r=-0.89 ; p<0.05$ ) (Fig. $6 C)$. After recovery from motor symptoms, 5-HT concentrations returned to levels similar to those at baseline (Fig. 4A). This decrease was positively correlated with recovery time $(r=0.92 ; p<0.05)$ (Fig. $6 D)$ and with the level of dopaminergic denervation, estimated by counts of TH- or DAT-positive fibers $(r=0.97, p<0.01 ; r=0.89, p<0.05$, respectively) (Fig. $6 \mathrm{~F}$ ).

Effects on 5HIAA levels

The expression of motor symptoms was accompanied by similar changes in 5HIAA concentration in both striatal territories, and recovery was also very similar. In the motor symptom state, 5HIAA levels were approximately half those in the normal state, for both the sensorimotor and associative-limbic striatum $(62.4 \pm 9.6$ and $71.3 \pm 12.5 \%$, respectively; $p<0.01)$ (Fig. $4 B$ ). After recovery from motor symptoms, 5HIAA levels were significantly higher than those in the normal and motor symptom state $(p<0.01)$ (Fig. 4B).

\section{Effects on 5HIAA/5-HT ratio}

A large decrease in the 5HIAA/5-HT ratio was observed in the sensorimotor striatum $(p<0.01)$ (Fig. $4 C$ ) during the symptomatic state. After recovery, this ratio remained abnormally low, given the increase in 5HIAA levels described above $(p<0.01)$ (Fig. 4C). A decrease in the 5HIAA/5-HT ratio was also observed
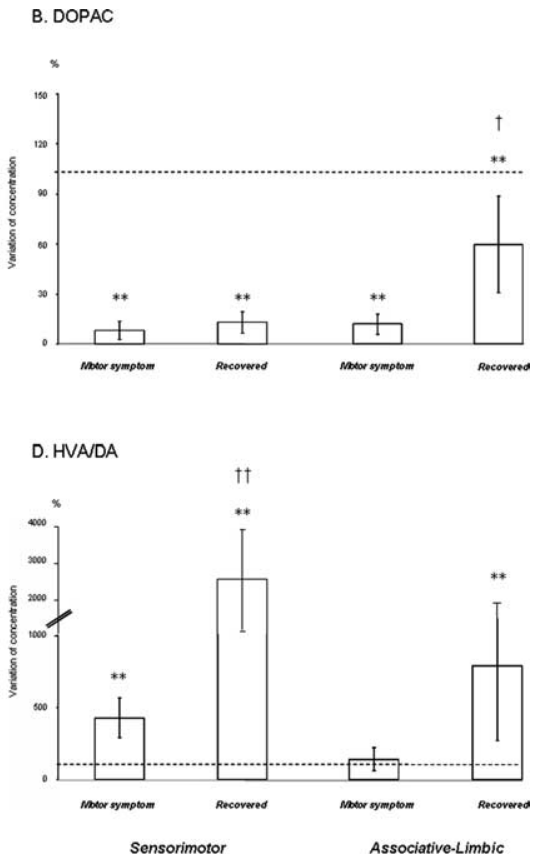

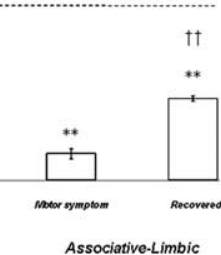

DOPAC ( $\boldsymbol{B})$, HVA $(\boldsymbol{C})$ concentrations, and HVA/DA ratio $(\boldsymbol{D})$ in
normal, symptomatic, and recovery states. Results for the five sensorimotor and associative/limbic striatal territories during the normal, symptomatic, and red by a vertical bar. All of the concen-
monkeys (mean \pm SEM) are shown for the motor symptom and recovery states, represented trations are expressed as a percentage of basal levels (set at 100\%) which are indicated by a dotted line. ${ }^{* *} p<0.01$ versus the normal state; ${ }^{\dagger} p<0.05,{ }^{\dagger \dagger} p<0.01$ versus the motor symptom state.

in the associative-limbic territory during motor symptom expression, but this decrease was smaller than that in the sensorimotor striatum. Nonetheless, this decrease was positively correlated with motor symptom expression $(r=0.89 ; p<0.05)$. Recovery from motor symptoms was associated with a large increase in the 5HIAA/5-HT ratio in the associative-limbic territory, to levels higher than those in the normal and motor symptom state $(p<0.01)$ (Fig. $4 C$ ). The HIAA/5-HT ratio was found to be positively correlated with the number of 5-HT fibers present in the associative-limbic territory after recovery $(r=0.95 ; p<$ 0.05) (Fig. 6E).

\section{Effects on GABA and Glu levels}

Changes in GABA and Glu concentrations during the expression of motor symptoms and after recovery were similar. In the sensorimotor territory, GABA and Glu concentrations were both increased during the motor symptom state $(p<0.01)$ (Fig. $5 A, B)$. Furthermore, a negative correlation was found between the intensity of motor symptom expression and changes in GABA concentration $(r=-0.89 ; p<0.05)$ (Fig. 6A). After motor recovery, mean GABA and Glu concentrations did not differ significantly from those in the motor symptom state, remaining higher than those in the normal state $(p<0.01)$ (Fig. $5 A, B)$. In the associative-limbic territory, GABA concentration changed little during the expression of motor symptoms, whereas Glu concentration increased slightly (Fig. 5A,B). After recovery, Glu concentration returned to normal levels, whereas GABA concentration fell to values significantly lower than at baseline $(-48.3 \pm$ 9.3\%; $p<0.01$ ) (Fig. 5A,B).

\section{Discussion}

We compared neurochemical changes in two striatal territories and three states (normal, motor symptom expression after MPTP injection and recovery) by repeated intracerebral microdialysis in 
A. Serotonin

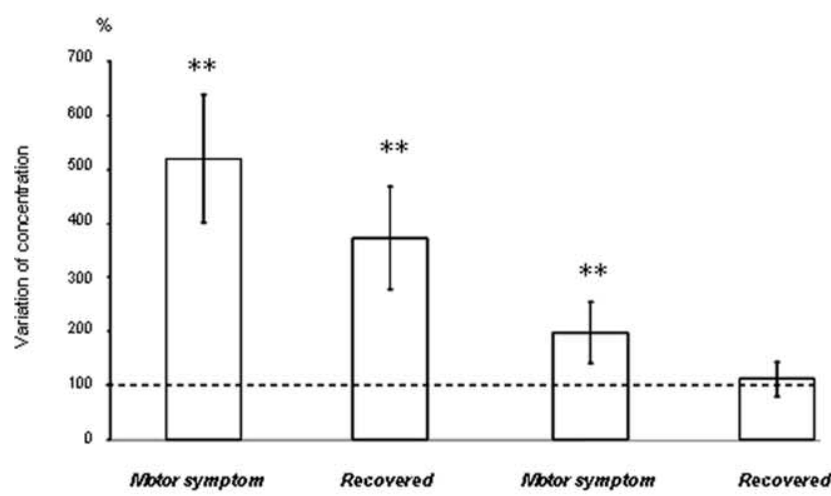

B. 5 HIAA

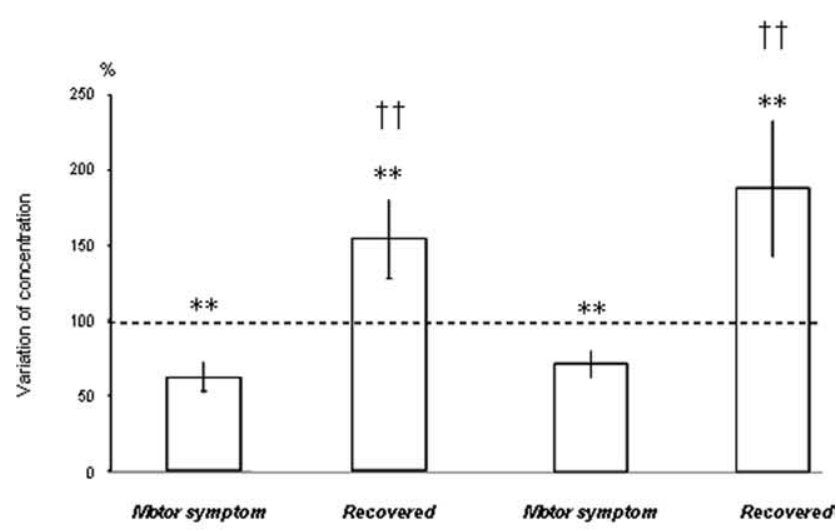

C. $5 \mathrm{HIAA} 5 \mathrm{HT}$

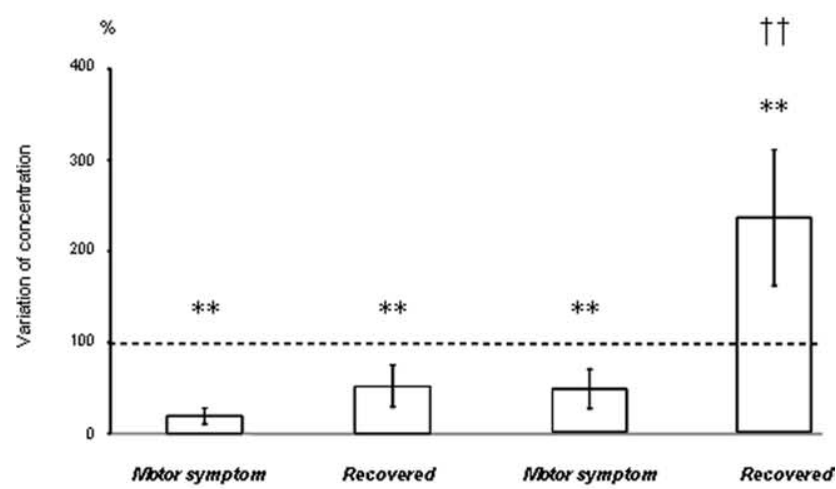

Sensorimotor

Associative-Limbic

Figure 4. $\quad \boldsymbol{A}-\boldsymbol{C}$, Schematic representation of serotonin $(\boldsymbol{A})$ and $5 \mathrm{HIAA}(\boldsymbol{B})$ concentrations and 5HIAA/5-HT ratio $(C)$ in sensorimotor and associative-limbic striatal territories during the nor$\mathrm{mal}$, symptomatic, and recovery states. Results for the five monkeys (mean \pm SEM) are shown for the motor symptom and recovery states, represented by a vertical bar. All concentrations are expressed as a percentage of basal levels (set at 100\%) which are represented by a dotted line. ${ }^{* *} p<0.01$ versus the normal state; ${ }^{{ }^{\dagger+}} p<0.01$ versus the motor symptom state.

awake monkeys. The symptomatic state was associated with a large decrease in DA concentration and a large increase in 5-HT, Glu, and GABA levels in the sensorimotor striatum. DA levels decreased less sharply in the associative-limbic striatum, whereas

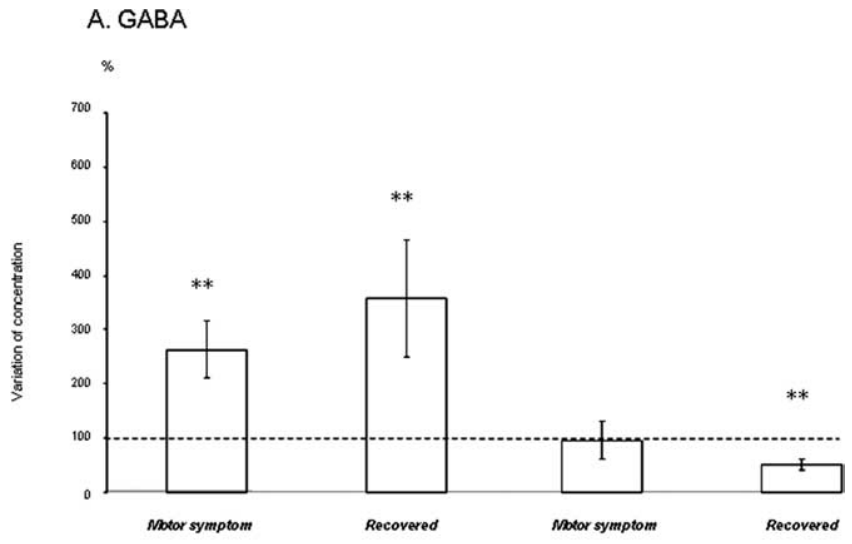

B. Glutamate

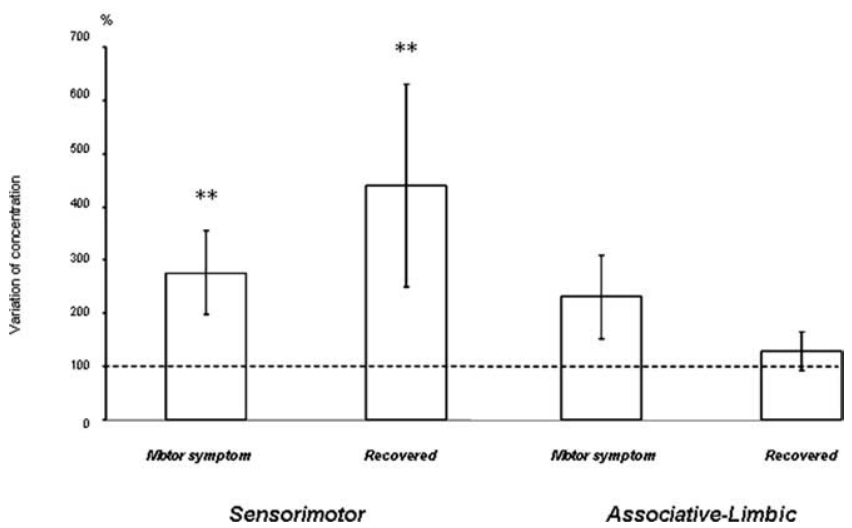

Figure 5. $\quad \boldsymbol{A}, \boldsymbol{B}$, Schematic representation of GABA $(\boldsymbol{A})$ and glutamate $(\boldsymbol{B})$ concentrations in sensorimotor and associative-limbic striatal territories during the normal, symptomatic, and recovery states. Results for the five monkeys (mean $\pm S E M$ ) are shown for the motor symptom and recovery states, represented by a vertical bar. All concentrations are expressed as a percentage of the basal level (set at $100 \%$ ) which is represented by a dotted line. ${ }^{* *} p<0.01$ versus the normal state.

5-HT and Glu levels increased (GABA levels were unaffected). DA concentration was increased after complete recovery, particularly in the associative-limbic territory, with a higher turnover (as reflected by the HVA/DA ratios) in both territories. 5-HT metabolite (5HIAA) levels increased in both striatal territories, whereas 5-HT turnover increased in the associative-limbic territory only. Glu and GABA levels remained high in the sensorimotor territory.

\section{Relevance of repeated microdialysis for investigating neurochemical modifications}

Repeated microdialysis has been performed in anesthetized (Wang et al., 1990; Kolachana et al., 1994) and awake monkeys (Davis et al., 1997), demonstrating the long-term viability of microdialysis preparations in primates (Bradberry, 2000). Consistent with this, neurotransmitter concentrations in our study did not differ significantly between the third and supplementary dialyses, except for DOPAC and Glu. The three dialysis sessions induced moderate cellular damage, with a limited glial reaction around the probe tract, as in rats (Georgieva et al., 1993). This approach appears to be accurate, with heterogeneous changes in DA concentration in different striatal territories reflecting the heterogeneity of dopaminergic degeneration reported for monkeys (Jan et al., 2000, 2003) and humans (Kish et al., 1988). 


\section{Modifications associated with motor symptom expression}

We observed a massive decrease in striatal DA, DOPAC, and HVA concentrations, as reported in parkinsonian patients postmortem (Hornykiewicz, 1975; Agid et al., 1987; Calon et al., 2003) and MPTP monkeys (Elsworth et al., 1987, 2000; Pifl et al., 1991). These decreases are consistent with major dopaminergic fiber losses, greater in the sensorimotor than in the associativelimbic territory (Mounayar et al., 2007). The HVA/DA and DOPAC/DA ratios increased in the sensorimotor striatum only. This may reflect rapid compensation involving increases in synthesis, release, and metabolism in surviving nigrostriatal DA neurons (Dentressangle et al., 2001).

The observed increases in Glu and GABA levels are consistent with the high Glu and GABA concentrations in the striatum of PD patients (Kish et al., 1987; Hornykiewicz, 2001) and increases in striatal Glu (Lindefors and Ungerstedt, 1990; Meshul et al., 1999) and GABA levels (Lindefors, 1993; Bruet et al., 2003) reported in 6-OHDA rats. Glu concentration increases could be attributable to an increase in glutamatergic corticostriatal input (Kornhuber and Kornhuber, 1986) via the vesicular Glu 1 transporter, whereas thalamostriatal input is unaffected (Raju et al., 2008). Changes in GABAergic neurotransmission in PD patients and 6-OHDA rats suggest that PD motor sign expression depends on increases in striatal GABAergic neurotransmission. However, we found a negative correlation between GABA concentration in the sensorimotor striatum and the motor score. Striatal GABA may originate from two populations of neurons: medium spiny neurons expressing local and close axonal collaterals and interneurons strongly inhibiting striatal neuron projections (Plenz, 2003). These interneurons seem to generate a strong inhibitory feedback, decreasing the tendency of striatal output activity to increase attributable to increases in Glu release. This may lead to rapid compensation and milder motor symptoms.

The few studies addressing changes in serotoninergic neurotransmission in cases of dopaminergic depletion have generated conflicting conclusions, with 5-HT concentrations reportedly decreasing (Pérez-Otaño et al., 1991), remaining stable (Pifl et al., 1991), or increasing (Schneider, 1990) in MPTP monkeys. Decreases in 5-HT concentration have been reported in parkinsonian patients (Scatton et al., 1983; Ohara et al., 1998), but this may have been attributable to L-DOPA treatment. We observed an increase in 5-HT concentration which was greater in the sensorimotor than in the associative-limbic striatum. Ultrastructural studies on 6-OHDA rats (Zhou et al., 1991), MPTP mice (Yamazoe et al., 2001), and MPTP monkeys (Gaspar et al., 1993) have demonstrated that nigrostriatal dopaminergic pathway destruction leads to serotoninergic hyperinnervation of the striatum. These data are consistent with our neurochemical findings and with the negative correlation between $\mathrm{TH}$-positive fiber counts and 5-HT levels in the associative-limbic striatum. We

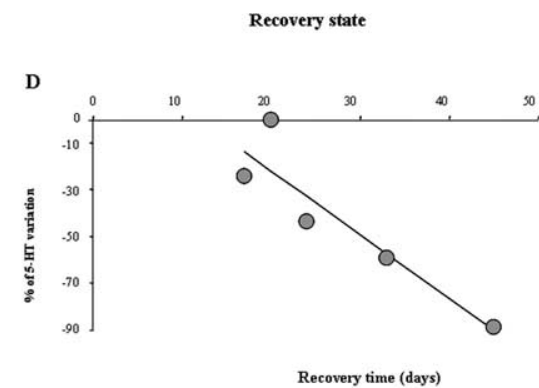

E

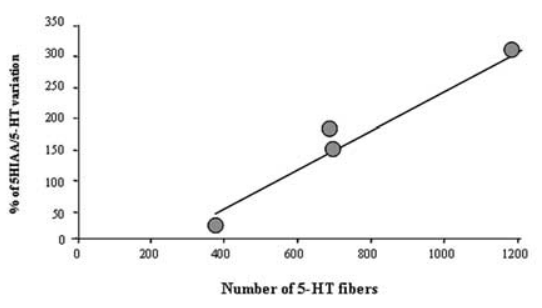

F

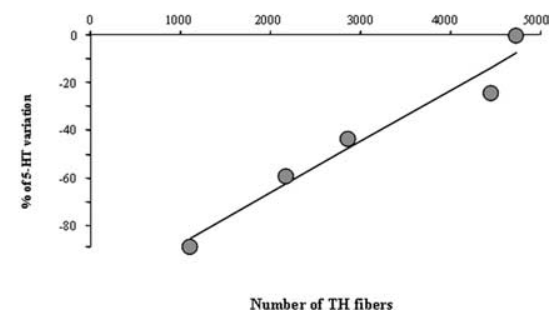

Figure 6. $\boldsymbol{A}-\boldsymbol{F}$, Correlation between neurochemistry and behavior $(\boldsymbol{A}, \boldsymbol{D})$, dopaminergic $(\boldsymbol{B}, \boldsymbol{C}, \boldsymbol{F})$, and serotoninergic denervation $(\boldsymbol{E})$ in the sensorimotor $(\boldsymbol{A}-\boldsymbol{C})$ and associative-limbic $(\boldsymbol{D}-\boldsymbol{F})$ territories. $\boldsymbol{A}, r=-0.89, p<0.05 ; \boldsymbol{B}, r=0.95, p<0.05 ; \boldsymbol{C}$, $r=-0.89, p<0.05 ; \boldsymbol{D}, r=-0.92, p<0.05 ; \boldsymbol{E}, r=0.98, p<0.01 ; \boldsymbol{F}, r=0.98, p<0.01$.

also observed a decrease in 5HIAA levels and the 5HIAA/5-HT ratio in both striatal territories, reflecting a slowing of 5-HT metabolism, as described previously in MPTP monkeys (Schneider, 1990).

\section{Modifications associated with motor function recovery}

Until recently, compensatory mechanisms associated with recovery from PD motor symptoms were thought to result purely from the adaptive properties of dopaminergic neurons (Zigmond, 1997). Studies on MPTP monkeys with motor symptoms of varying degrees of severity have shown that a limited DA loss can be counterbalanced by an increase in DA release from the remaining fibers. With more extensive DA fiber loss, DA release becomes insufficient, and substantial changes in DA metabolism, as reflected by the HVA/DA ratio, are required (Elsworth et al., 1989, 2000; Rose et al., 1989). However, normal motor performance is recovered if striatal DA levels reach $5-10 \%$ of those in the normal state (Elsworth et al., 2000). Our results are consistent with these observations. We observed recovery despite considerable striatal DA depletion. This recovery was accompanied by increases in DA, HVA, and DOPAC levels and DOPAC/DA and HVA/DA ratios throughout the striatum. Various mechanisms may account for this slight increase in DA levels, facilitating recovery from symptoms: (1) an increase in DA release from the remaining fibers, attributable to an increase in the glutamatergic input of the SNc (Bezard et al., 1997); (2) the sprouting of new fibers from 
the remaining terminals (Elsworth et al., 2000), supported by morphological data from 6-OHDA rats (Blanchard et al., 1996) and MPTP monkeys (Song and Haber, 2000) and by higher THand DAT-positive fiber counts in monkeys that recovered than in monkeys with stable motor symptoms (Mounayar et al., 2007); and (3) increased DA diffusion from release sites. The latter phenomenon has been reported in spontaneously recovering MPTP cats (Schneider et al., 1994) and rats with partial 6-OHDA lesions (Dentressangle et al., 2001). This diffusion could be facilitated by the volume transmission (Fuxe and Agnati, 1991) promoted under DA depletion (Robinson and Whishaw, 1988) and by a decrease in the number of DA reuptake sites in the striatum (Bezard et al., 2000, 2001). We found that associative-limbic DA levels after recovery were similar to those in the normal state. Thus, DA may diffuse from this relatively spared part of the striatum to nearby denervated parts, increasing DA concentrations to supraliminar levels, allowing functional recovery.

Neurochemical compensatory mechanisms extrinsic to the dopaminergic system have been little studied. We provide the first demonstration of changes in extracellular striatal Glu and GABA concentrations associated with PD motor symptom recovery. Glu or GABA concentrations did not differ during the motor symptom and recovery states, except GABA levels which were significantly lower in the associative-limbic striatum during recovery. Thus, normal striatal Glu and GABA concentrations are not required for complete motor recovery, suggesting that these two neurotransmission systems do not play a major role in recovery. The lack of correlation between levels of neurotransmitters and recovery time is consistent with this interpretation.

We observed several changes in striatal 5-HT neurotransmission after recovery. 5-HT concentration returned to basal levels in the associative-limbic striatum. 5-HIAA levels were higher than those in the normal and motor symptom states, in both the associative-limbic and sensorimotor territories, whereas the increase in the 5HIAA/5-HT ratio was limited to the associativelimbic territory. Our data are entirely consistent with striatal 5-HT neurotransmission and 5-HT metabolism, in particular, being involved in recovery mechanisms, as previously suggested (Schneider and Rothblat, 1991). This interpretation is supported by the stronger 5-HT labeling observed in the striatal functional territories of recovered monkeys and weaker labeling observed in monkeys with stable motor symptoms than in normal monkeys (Mounayar et al., 2007). This serotoninergic hyperinnervation, often observed in animal models of PD (Zhou et al., 1991; Gaspar et al., 1993; Yamazoe et al., 2001), may be compensatory. Indeed, 5-HT may promote DA release from the remaining dopaminergic fibers (Benloucif and Galloway, 1991; Yadid et al., 1994). Finally, the correlation between 5-HT concentration in the associative-limbic territory and recovery time strongly suggests a role for 5-HT in recovery.

In conclusion, this study confirms the importance of striatal dopaminergic neurotransmission and metabolism for recovery from symptoms in this monkey model with MPTP-induced lesions. Our results suggest that "a minimum threshold" of striatal DA is required to support striatal functions. They also show that glutamatergic and GABAergic neurotransmission plays only a minor role in striatal recovery. Our findings clearly implicate 5-HT and its metabolism in compensatory mechanisms and demonstrate that the associative-limbic striatal territory, preserved both in this animal model and in PD, plays an important role in recovery mechanisms. We now need to determine whether the recovery process in our model is equivalent to the compensatory mechanisms operating during the presymptomatic stage of natural disease. This could allow the development of new therapeutic strategies effective in the early phase of the disease.

\section{References}

Agid Y, Javoy-Agid F, Ruberg M (1987) Biochemistry of neurotransmitters in Parkinson's disease. In: Movement Disorders (Marsden CD, Fahn S, eds), pp 166-230. London: Springer/Butterworth.

Benloucif S, Galloway MP (1991) Facilitation of dopamine release in-vivo by serotonin agonists: studies with microdialysis. Eur J Pharmacol 200:1-8.

Bernheimer H, Birkmayer W, Hornykiewicz O, Jellinger K, Seitelberger F (1973) Brain dopamine and the syndromes of Parkinson and Huntington. Clinical, morphological and neurochemical correlations. J Neurol Sci 20:415-455.

Bezard E, Boraud T, Bioulac B, Gross CE (1997) Compensatory effects of glutamatergic inputs to the substantia nigra pars compacta in experimental parkinsonism. Neuroscience 81:399-404.

Bezard E, Jaber M, Gonon F, Boireau A, Bloch B, Gross CE (2000) Adaptative changes in the nigrostriatal pathway in response to increased 1-methyl-4-phenyl-1,2,3,6-tetrahydropyridine-induced neurodegeneration in the mouse. Eur J Neurosci 12:2892-2900.

Bezard E, Dovero S, Prunier C, Ravenscroft P, Chalon S, Guilloteau D, Crossman AR, Bioulac B, Brotchie JM, Gross CE (2001) Relationship between the appearance of symptoms and the level of nigrostriatal degeneration in a progressive 1-methyl-4-phenyl-1,2,3,6-tetrahydropyridine-lesioned macaque model of Parkinson's disease. J Neurosci 21:6853-6861.

Blanchard V, Anglade P, Dziewczapolski G, Savasta M, Agid Y, RaismanVozari R (1996) Dopaminergic sprouting in the rat striatum after partial lesion of the substantia nigra. Brain Res 709:319-325.

Bradberry CW (2000) Applications of microdialysis methodology in nonhuman primates: Practice and rationale. Crit Rev Neurobiol 14:143-163.

Bruet N, Windels F, Carcenac C, Feuerstein C, Bertrand A, Poupard A, Savasta M (2003) Neurochemical mechanisms induced by high frequency stimulation of the subthalamic nucleus: increase of extracellular striatal glutamate and GABA in normal and hemiparkinsonian rats. J Neuropathol Exp Neurol 62:1228-1240.

Calon F, Morissette M, Rajput AH, Hornykiewicz O, Bédard PJ, Di Paolo T (2003) Changes of GABA receptors and dopamine turnover in the postmortem brains of parkinsonian with levodopa-induced motor complications. Mov Disord 18:241-253.

Davis MD, Heffner TG, Cooke LW (1997) Dopamine agonist-induced inhibition of neurotransmitter release from the awake squirrel monkey putamen as measured by microdialysis. J Neurochem 68:659-666.

de Lange EC, de Boer AG, Breimer DD (2000) Methodological issues in microdialysis sampling for pharmacokinetic studies. Adv Drug Deliv Rev 45:125-148.

Dentresangle C, Le Cavorsin M, Savasta M, Leviel V (2001) Increased basal extracellular dopamine levels and normal evoked dopamine overflow at an early stage of striatal deafferentation. Brain Res 893:178-185.

Elsworth JD, Deutch AY, Redmond DE Jr, Sladek JR Jr, Roth RH (1987) Effects of 1-methyl-4-phenyl-1,2,3,6-tetrahydropyridine (MPTP) on catecholamines and metabolites in primate brain and CSF. Brain Res 415:293-299.

Elsworth JD, Deutch AY, Redmond DE Jr, Taylor JR, Sladek JR Jr, Roth RH (1989) Symptomatic and asymptomatic 1-methyl-4-phenyl-1,2,3,6tetrahydropyridine treated primates: biochemical changes in striatal regions. Neuroscience 33:323-331.

Elsworth JD, Taylor JR, Sladek JR Jr, Collier TJ, Redmond DE Jr, Roth RH (2000) Striatal dopaminergic correlates of stable parkinsonism and degree of recovery in old-world primates one year after MPTP treatment. Neuroscience 95:399-408.

François C, Yelnik J, Tandé D, Agid Y, Hirsch EC (1999) Dopaminergic cell group A8 in the monkey: anatomical organization and projections to the striatum. J Comp Neurol 414:334-347.

Fuxe K, Agnati LF (1991) Two principal modes of electrochemical communication in the brain: volume versus wiring transmission. In: Volume transmission in the brain: novel mechanisms for neural transmission (Fuxe K, Agnati LF, eds), pp 1-9. New York: Raven.

Gaspar P, Febvret A, Colombo J (1993) Serotoninergic sprouting in primate MPTP-induced hemiparkinsonism. Exp Brain Res 96:100-106.

Georgieva J, Luthman J, Mohringe B, Magnusson O (1993) Tissue and mi- 
crodialysate changes after repeated and permanent probe implantation in the striatum of freely moving rats. Brain Res Bull 31:463-470.

Hefti F, Melamed E, Wurtman RJ (1980) Partial lesions of the dopaminergic nigro-striatal system in rat brain: biochemical characterization. Brain Res 195:123-137.

Hornykiewicz O (1975) Brain monoamines and parkinsonism. Natl Inst Drug Abuse Res Monogr Ser 3:13-21.

Hornykiewicz O (1998) Biochemical aspects of Parkinson's disease. Neurology $51:$ :S2-S9.

Hornykiewicz O (2001) Chemical neuroanatomy of the basal ganglia - nor$\mathrm{mal}$ and in Parkinson's disease. J Chem Neuroanat 22:3-12.

Jan C, François C, Tandé D, Yelnik J, Tremblay L, Agid Y, Hirsch E (2000) Dopaminergic innervation of the pallidum in the normal state, MPTPtreated monkeys and Parkinsonian patients. Eur J Neurosci 12:4525-4535.

Jan C, Pessiglione M, Tremblay L, Tandé D, Hirsch EC, François C (2003) Quantitative analysis of dopaminergic loss in relation to functional territories in MPTP-treated monkeys. Eur J Neurosci 18:2082-2086.

Khan SH, Shuaib A (2001) The technique of intracerebral microdialysis. Methods 23:3-9.

Kish S, Rajput A, Gilbert J, Rozdilsky B, Chang LJ, Shannak K, Hornykiewicz O (1987) GABA-dopamine relationship in Parkinson's disease striatum. Adv Neurol 45:75-77.

Kish SJ, Shannak K, Hornykiewicz O (1988) Uneven pattern of dopamine loss in the striatum of patients with idiopathic Parkinson's disease. Pathophysiologic and clinical implications. N Engl J Med 318:876-880.

Kolachana BS, Saunders RC, Weinberger DR (1994) An improved methodology for routine in vivo microdialysis in non-human primates. J Neurosci Methods 55:1-6.

Kornhuber J, Kornhuber ME (1986) Presynaptic dopaminergic modulation of cortical input to the striatum. Life Sci 39:699-674.

Lindefors N (1993) Dopaminergic regulation of glutamic acid decarboxylase mRNA expression and GABA release in the striatum: a review. Prog Neuropsychopharmacol Biol Psychiatry 17:887-903.

Lindefors N, Ungerstedt U (1990) Bilateral regulation of glutamate tissue and extracellular levels in caudate-putamen by midbrain dopamine neurons. Neurosci Lett 115:248-252.

McCallum SE, Parameswaran N, Perez XA, Bao S, McIntosh JM, Grady SR, Quik M (2006) Compensation in pre-synaptic dopaminergic function following nigrostriatal damage in primates. J Neurochem 96:960-972.

Meshul CK, Emre N, Nakamura CM, Allen C, Donohue MK, Buckman JF (1999) Time-dependent changes in striatal glutamate synapses following a 6-hydroxydopamine lesion. Neuroscience 88:1-16.

Mounayar S, Boulet S, Tandé D, Jan C, Pessiglione M, Hirsch EC, Féger J, Savasta M, François C, Tremblay L (2007) A new model to study compensatory mechanisms in MPTP-treated monkeys exhibiting recovery. Brain 130:2898-2914.

Ohara K, Kondo N, Ohara K (1998) Changes of monoamines in postmortem brains from patients with diffuse Lewy body disease. Prog Neuropsychopharmacol Biol Psychiatry 22:311-317.

Pérez-Otaño I, Herrero MT, Oset C, De Ceballos ML, Luquin MR, Obeso JA, Del Río J (1991) Extensive loss of brain dopamine and serotonin induced by chronic administration of MPTP in the marmoset. Brain Res 567:127-132.

Petzinger GM, Fisher B, Hogg E, Abernathy A, Arevalo P, Nixon K, Jakowec MW (2006) Behavioural motor recovery in the MPTP lesioned squirrel monkey: changes in striatal dopamine and expression of tyrosine hydroxylase and dopamine transporter proteins. J Neurosci Res 83:332-347.

Pifl C, Schingnitz G, Hornykiewicz O (1991) Effect of MPTP on the regional distribution of brain monoamines in the rhesus monkey. Neuroscience 44:591-605.
Plenz D (2003) When inhibition goes incognito: feedback interaction between spiny projection neurons in striatal function. TRENDS Neurosci 26:436-443.

Raju DV, Ahern TH, Shah DJ, Wright TM, Standaert DG, Hall RA, Smith Y (2008) Differential synaptic plasticity of the corticostriatal and thalamostriatal systems in an MPTP-treated monkey model of parkinsonism. Eur J Neurosci 27:1647-1658.

Robinson S, Freeman P, Moore C, Touchon JC, Krentz L, Meshul CK (2003) Acute and subchronic MPTP administration differentially affects striatal glutamate synaptic function. Exp Neurol 180:74-87.

Robinson TE, Whishaw IQ (1988) Normalization of extracellular dopamine in the striatum following recovery from a partial unilateral 6-OHDA lesion of the substantia nigra: a microdialysis study in freely moving rats. Brain Res 450:209-224.

Rose S, Nomoto M, Kelly E, Kilpatrick G, Jenner P, Marsden CD (1989) Increased caudate dopamine turnover may contribute to the recovery of motor function in marmosets treated with the dopaminergic neurotoxin MPTP. Neurosci Lett 101:305-310.

Scatton B, Javoy-Agid F, Rouquier L, Dubois B, Agid Y (1983) Reduction of cortical dopamine, noradrenaline, serotonin and their metabolites in Parkinson's disease. Brain Res 275:321-328.

Schneider JS (1990) Chronic exposure to low doses of MPTP. II. Neurochemical and pathological consequences in cognitively-impaired, motor asymptomatic monkeys. Brain Res 534:25-36.

Schneider JS, Kovelowski CJ 2nd (1990) Chronic exposure to low doses of MPTP. I. Cognitive deficits in motor asymptomatic monkeys. Brain Res 519:122-128.

Schneider JS, Rothblat DS (1991) Neurochemical evaluation of the striatum in symptomatic and recovered MPTP-treated cats. Neuroscience 44:421-429.

Schneider JS, Rothblat DS, DiStefano L (1994) Volume transmission of dopamine over large distances may contribute to recovery from experimental parkinsonism. Brain Res 643:86-91.

Schwarting RK, Huston JP (1996) Unilateral 6-hydroxydopamine lesions of meso-striatal dopamine neurons and their physiological sequelae. Prog Neurobiol 49:215-266.

Skirboll S, Wang J, Mefford I, Hsiao J, Bankiewicz KS (1990) In vivo changes of catecholamines in hemiparkinsonian monkeys measured by microdialysis. Exp Neurol 110:187-193.

Song DD, Haber SN (2000) Striatal responses to partial dopaminergic lesion: evidence for compensatory sprouting. J Neurosci 20:5102-5114.

Tossman U, Ungerstedt U (1986) Microdialysis in the study of extracellular levels of amino acids in the rat brain. Acta Physiol Scand 128:9-14.

Wang J, Skirboll S, Aigner TG, Saunders RC, Hsiao J, Bankiewicz KS (1990) Methodology of microdialysis of neostriatum in hemiparkinsonian nonhuman primates. Exp Neurol 110:181-186.

Yadid G, Pacak K, Kopin IJ, Goldstein DS (1994) Endogenous serotonin stimulates striatal dopamine release in conscious rats. J Pharmacol Exp Ther 270:1158-1165.

Yamazoe I, Takeuchi Y, Matsushita H, Kawano H, Sawada T (2001) Serotonergic heterotypic sprouting in the unilaterally dopamine-depleted mouse neostriatum. Dev Neurosci 23:78-83.

Zhou FC, Bledsoe S, Murphy J (1991) Serotoninergic sprouting is induced by dopamine-lesion in substantia nigra of adult rat brain. Brain Res 556:108-116.

Zigmond MJ (1997) Do compensatory processes underlie the preclinical phase of neurodegenerative disease? Insights from an animal model of parkinsonism. Neurobiol Dis 4:247-253.

Zigmond MJ, Abercrombie ED, Berger TW, Grace AA, Stricker EM (1990) Compensations after lesions of central dopaminergic neurons: some clinical and basic implications. Trends Neurosci 13:290-296. 